\title{
Targeting of RGS7/G $\beta 5$ to the Dendritic Tips of ON-Bipolar Cells Is Independent of Its Association with Membrane Anchor R7BP
}

\author{
Yan Cao, ${ }^{1}$ Hongman Song, ${ }^{2}$ Haruhisa Okawa, ${ }^{4}$ Alapakkam P. Sampath,,${ }^{4,5}$ Maxim Sokolov, ${ }^{2,3}$ and \\ Kirill A. Martemyanov ${ }^{1}$ \\ ${ }^{1}$ Department of Pharmacology, University of Minnesota, Minneapolis, Minnesota 55455, Departments of ${ }^{2}$ Biochemistry and ${ }^{3}$ Ophthalmology, West Virginia \\ University Eye Institute, West Virginia University School of Medicine, Morgantown, West Virginia 26506, and ${ }^{4}$ Neuroscience Graduate Program, ${ }^{5}$ Zilkha \\ Neurogenetic Institute, and ${ }^{6}$ Department of Physiology and Biophysics, University of Southern California, Los Angeles, California 90089
}

Complexes of regulator of G-protein signaling (RGS) proteins with G-protein $\beta 5(\mathrm{G} \beta 5)$ subunits are essential components of signaling pathways that regulate the temporal characteristics of light-evoked responses in vertebrate retinal photoreceptors and 0N-bipolar cells. Recent studies have found that RGS/G $\beta 5$ complexes bind to a new family of adapter proteins, R9AP (RGS9 anchor protein) and R7 family binding protein (R7BP), that in case of the RGS9/G $\beta 5$ complex were shown to determine its precise subcellular targeting to either the outer segment of photoreceptors or postsynaptic structures of striatal neurons, respectively. In this study, we establish that another trimeric complex consisting of RGS7, G $\beta 5$, and R7BP subunits is specifically targeted to the dendritic tips of retinal bipolar cells. However, examination of the mechanisms of complex targeting in vivo surprisingly revealed that the delivery of RGS7/G $\beta 5$ to the dendrites of ON-bipolar cells occurs independently of its association with R7BP. These findings provide a new mechanism for adapter-independent targeting of RGS/G $\beta 5$ complexes.

Key words: G-protein; signal transduction; RGS proteins; retina; intracellular targeting; knock-out mice

\section{Introduction}

G-protein signaling pathways play crucial roles for light reception in the vertebrate retina (Rodieck, 1998). In retinal photoreceptors, the stimulation of the G-protein-coupled receptor rhodopsin (Rh) by light triggers GTP binding to the G-protein transducin. Transducin promotes the hydrolysis of the second messenger cGMP, leading to membrane hyperpolarization and the suppression of neurotransmitter glutamate release (for review, see Burns and Arshavsky, 2005). The high temporal resolution of vision is achieved by rapid shutoff of the phototransduction cascade returning photoreceptor to the resting state and allowing the cell to generate responses to stimuli that are closely spaced in time (Chen, 2005). Recent studies have shown that the rate-limiting step in the recovery of photoreceptors from light excitation is the inactivation of transducin (Krispel et al., 2006). Transducin inactivation is catalyzed by regulator of G-protein signaling (RGS) protein complex that consists of a scaffold

Received July 10, 2008; revised Aug. 31, 2008; accepted Sept. 11, 2008

This work was supported by National Institutes of Health (NIH) Grant EY018139 (K.A.M.), NIH/National Center for Research Resources 2P20 RR15574 (enter of Biomedical Research Excellence in Sensory Neuroscience (M.S.), NIH Grant EY017606 (A.P.S.), and a Karl Kirchgessner Foundation Vision Research Grant (A.P.S.). We thank Dr. William Simonds (NIH) for the generous gift of anti-RGS7, anti-G $\beta 5$, and anti-R7BP antibodies and Dr. Jason Chen (Virginia Commonwealth University, Richmond, VA) for providing $G \beta 5$ knock-out mice. We also thank Gilbert Ahlstrend and Mark Sanders at the Imaging Center, University of Minnesota, for the help with retina plastic sections.

Correspondence should be addressed to Dr. Kirill Martemyanov, Department of Pharmacology, University of Minnesota, 6-120 Jackson Hall, 321 Church Street SE, Minneapolis, MN 55455. E-mail: martemyanov@umn.edu. D01:10.1523/JNEUROSCI.3282-08.2008

Copyright $\odot 2008$ Society for Neuroscience $\quad 0270-6474 / 08 / 2810443-07 \$ 15.00 / 0$
[G-protein $\beta 5$ (Gß5)], a catalytic molecule (RGS9-1), and a targeting subunit [RGS9 anchor protein (R9AP)] (Burns and Arshavsky, 2005). Genetic studies in mice revealed R9AP to be indispensable for the delivery of the complex to the outer segment of photoreceptors, where all of the RGS9-1/G $\beta 5 /$ R9AP is normally found (Martemyanov et al., 2003).

G-protein-inactivating complexes containing G $\beta 5$ are also critical for the synaptic transmission between photoreceptors and ON-bipolar cells, because the elimination of G $\beta 5$ in mice causes the failure of ON-bipolar cells to relay the photoreceptor's excitation (Rao et al., 2007). The subunit composition of the G $\beta 5$ complexes in the inner retina differs from that of photoreceptors. Instead of a photoreceptor-specific RGS9-1, these complexes contain other members of the R7 RGS subfamily: RGS6, RGS7, or RGS11 (Song et al., 2007). Likewise, instead of R9AP, R7 RGS/ G $\beta 5$ complexes in the inner retina are bound to R7 RGS binding protein (R7BP) (Song et al., 2007), an R9AP homolog. Association of R7 RGS proteins with R7BP was also reported in the brain, where it was shown to be critical for both RGS expression and postsynaptic targeting (Anderson et al., 2007b).

Both RGS7/G $\beta 5$ and RGS11/G $\beta 5$ were reported to be enriched specifically in the tips of the ON-bipolar dendrites (Morgans et al., 2007; Rao et al., 2007), suggesting a role in the regulation of mGluR6-Go G-protein signaling cascade that mediates the responses of ON-bipolar cells to changes in glutamate released from photoreceptors. In this study, we report that R7BP colocalizes with RGS7/G $\beta 5$ at the postsynaptic sites of the ON- 
bipolar neurons. Analysis of the mechanisms underpinning the specific localization of the complex reveals that both expression and delivery of RGS7/G $\beta 5$ to the dendritic tips occur independently from R7BP. These data suggest a new mechanism for the postsynaptic targeting of R7 RGS proteins in the inner retina neurons as dimers with $G \beta 5$, as opposed to previously described adapter-directed mechanisms.

\section{Materials and Methods}

Antibodies and knock-out mice. The generation of rabbit anti-R9AP 144223 (Keresztes et al., 2004), sheep anti-RGS9c (Makino et al., 1999), and anti-phosducin (Sokolov et al., 2004) were described previously. Rabbit anti-RGS7 (7RC1), anti-R7BP (TRS), and anti-G $\beta 5$ (SGS) were generous gifts from Dr. William Simonds [National Institute of Diabetes and Digestive and Kidney Diseases/National Institutes of Health (NIH), Bethesda, MD]. Sheep anti-RGS6 antibodies were generated against amino acids 263-472 of mouse recombinant RGS6 (RGS6FL) and sheep antiRGS11 antibodies were raised against the C-terminal peptide: SPALQSTPREPAATSSPEGADGE. Commercial antibodies were as follows: mouse anti- $\beta$-actin (AC-15; Sigma), mouse anti-PKC $\alpha$ (ab11723; Abcam), and goat anti-mGluR6 (C18) and rabbit anti-G $\alpha$ o (K-20) from Santa Cruz Biotechnology.

The generation of R7BP (Anderson et al., 2007b), G $\beta 5$ (Chen et al., 2003), and R9AP (Keresztes et al., 2004) knock-outs has been described. RGS11-/- mice were generated by Lexicon Genetics and obtained from Mutant Mouse Regional Resource Centers. In these mice, the first four exons of RGS11 gene were targeted by homologous recombination to produce a null mutation as confirmed by the absence of RGS11 protein (see Fig. 2).

Serial sectioning and Western blotting. Serial tangential sectioning of the flat-mounted frozen rat retina was performed exactly as previously described (Song et al., 2007). Ten micrometer sections were collected and lysed in $50 \mu \mathrm{l}$ of sample buffer containing $125 \mathrm{~mm}$ Tris- $\mathrm{HCl}, \mathrm{pH}$ 6.8, 4\% SDS, and $6 \mathrm{~m}$ urea. Proteins were separated by SDS-PAGE, transferred onto a polyvinylidene difluoride membrane, and analyzed by Western blotting on an Odyssey Infrared Imaging System (LI-COR Biosciences).

Retina sections and immunohistochemistry. Dissected eyecups were fixed for 15 min with $4 \%$ paraformaldehyde, cryoprotected with $30 \%$ sucrose in PBS for $2 \mathrm{~h}$ at room temperature and embedded in OCT. Twelve micrometer frozen sections were blocked in PBS, $0.1 \%$ Triton $\mathrm{X}-100$, and $10 \%$ goat serum for $1 \mathrm{~h}$ and incubated with the primary antibody in PBS with $0.1 \%$ Triton and $2 \%$ goat serum for $2 \mathrm{~h}$ or overnight, washed four times with PBS, $1 \%$ Triton X-100 (PT2), and incubated with fluorophore-conjugated secondary antibodies in PT2 for $1 \mathrm{~h}$. For double staining, primary antibodies raised in the same species were directly labeled with Dylight-549 using a Microscale Antibody Labeling Kit (Thermo Fisher Scientific). Sections were washed three times for 5 min and mounted in Gel/Mount (Biomeda). Images were taken by Olympus Fluoview 1000 confocal microscope. For morphological analysis, $1.75 \mu \mathrm{m}$ sections of retinas embedded in Embed 812 were stained with $1 \%$ toluidine blue as described previously (Keresztes et al., 2004)

Subcellular fractionation. Retinas from one animal were lysed in 0.15 $\mathrm{ml}$ of PBS by homogenizing with a series of needles and were sedimented at $75,000 \times g$ for $30 \mathrm{~min}$. Supernatant containing cytoplasmic fraction was removed, and the pellet was resuspended and resedimented at $75,000 \times g$ for $30 \mathrm{~min}$. The resulting pellet was resuspended in $0.15 \mathrm{ml}$ of $\mathrm{PBS}$ and designated as the insoluble membrane fraction. Equal volume aliquots of supernatant and pellet fractions were mixed with the SDS sample buffer and subjected to SDS-PAGE analysis followed by Western blotting.

Electroretinography. Adult mice (1-3 months old) were dark adapted overnight and anesthetized with a $2 \%$ isoflurane $/ 50 \%$ oxygen mixture. The animal's pupils were dilated, and a disposable reference needle electrode (LKC Technologies) was inserted under the loose skin between the ears. Custom-made silver wire electrodes were positioned on the corneas. Ganzfeld flash electroretinography (ERG) recording was performed in UTAS-E4000 Visual Electrodiagnostic Test System using EMWIN 8.1.1 software (LKC Technologies), according to the manufacturer's protocols.

Single-cell recordings and light calibration. Whole-cell voltage-clamp recordings $\left(V_{\mathrm{m}}=-60 \mathrm{mV}\right)$ from rod bipolar cells in 200- $\mu \mathrm{m}$-thick dark-adapted retinal slices were made as described previously (Sampath et al., 2005). The internal solution for these experiments consisted of the following: $125 \mathrm{~K}$-aspartate, $10 \mathrm{KCl}, 10 \mathrm{HEPES}, 5 \mathrm{~N}$-methyl-D-glucamine (NMG)-hydroxyethylethylenediaminetriacetic acid, $0.5 \mathrm{CaCl}_{2}, 1 \mathrm{ATP}-$ $\mathrm{Mg}$, and 0.2 GTP-Mg; pH was adjusted to 7.2 with NMG-OH. Flash families were measured in response to a $10 \mathrm{~ms}$ flash from a blue lightemitting diode (LED) $\left(\lambda_{\max }, \sim 470 \mathrm{~nm}\right)$ whose strength initially generated a just-measurable response and increased by factors of 2 . Average responses per photon were calculated by averaging the response per photon across the dimmest responses of each cell [i.e., those between 5 and $25 \%$ of maximal amplitude (Sampath et al., 2005)].

To estimate the number of rhodopsin molecules activated per flash, we measured the light intensity of a $520 \mu \mathrm{m}$ spot focused on the preparation by the $20 \times 0.75$ numerical aperture (Nikon) condenser objective using a calibrated photodiode (United Detector Technologies). Light intensities were converted to equivalent $501 \mathrm{~nm}$ photons by convolving the powerscaled spectral output of the LED with the normalized spectral sensitivity curve for mouse Rh. The flash strengths were then converted to activated $\mathrm{Rh}$ per rod $\left(\mathrm{Rh}^{\star} / \mathrm{rod}\right)$ by estimating the collecting area of rod photoreceptors in the experimental setup. Dim flashes were delivered during suction electrode recordings from rod outer segments in clusters (Sampath et al., 2005), and the mean $\mathrm{Rh}^{\star} / \mathrm{rod}$ at each flash strength was determined from the scaling of the time-dependent variance to the mean response (Field and Rieke, 2002). Based on these factors, we estimated the rod collecting area in the experimental setup to be $0.18 \mu \mathrm{m}^{2}(n=6$ cells).

\section{Results \\ Colocalization of RGS7/G $\beta 5$ with R7BP in dendritic tips of ON-bipolar cells}

We used a high-affinity anti-R7BP antibody capable of detecting low levels of the protein in the retina (Nini et al., 2007) to compare the distribution pattern of R7BP with that of RGS7 using a serial sectioning approach. Data presented in Figure $1 \mathrm{~A}$ reveal that R7BP coincides with RGS7 in the same retinal sections that also contain $\mathrm{G} \alpha \mathrm{o}$, a G-protein mediating signal transmission between rods and ON-bipolar cells (Dhingra et al., 2000). Both $\mathrm{R} 7 \mathrm{BP}$ and RGS7 were found exclusively in the inner retina and did not show any overlap with RGS9-1 and R9AP in the photoreceptor layer.

Further analysis of RGS7 and R7BP distribution by immunohistochemical staining of retinal cross sections confirmed their colocalization in the bipolar cells as evidenced by overlap with the PKC $\alpha$-positive immunofluorescence in the outer plexiform layer (Fig. $1 B$ ). Close examination of immunostaining in this region revealed a punctate pattern characteristic of synaptic sites (Fig. $1 C)$. Indeed, both R7BP and RGS7 closely colocalized with mGluR6, indicating the presence of R7BP/RGS7 complexes in the dendritic tips of ON-bipolar neurons.

\section{Expression of R7 RGS proteins and R7BP in the retinas of knock-out mice lacking components of the complex}

To investigate the role of R7BP in mediating the postsynaptic localization and function of RGS7 in bipolar cells, we studied $\mathrm{R} 7 \mathrm{BP}-/-$ mice. Retinas of these mice had normal morphology and showed no signs of degeneration up to 4 months of age (Fig. $2 A$ ). Western blot analysis indicated that protein levels of RGS7, as well as other R7 RGS proteins and their binding partners, R9AP and G $\beta 5$, were unaffected by the R7BP elimination (Fig. $2 \mathrm{~B})$. This result is consistent with the similar lack of the R7BP effect on the expression of RGS7 in the striatum (Anderson et al., 

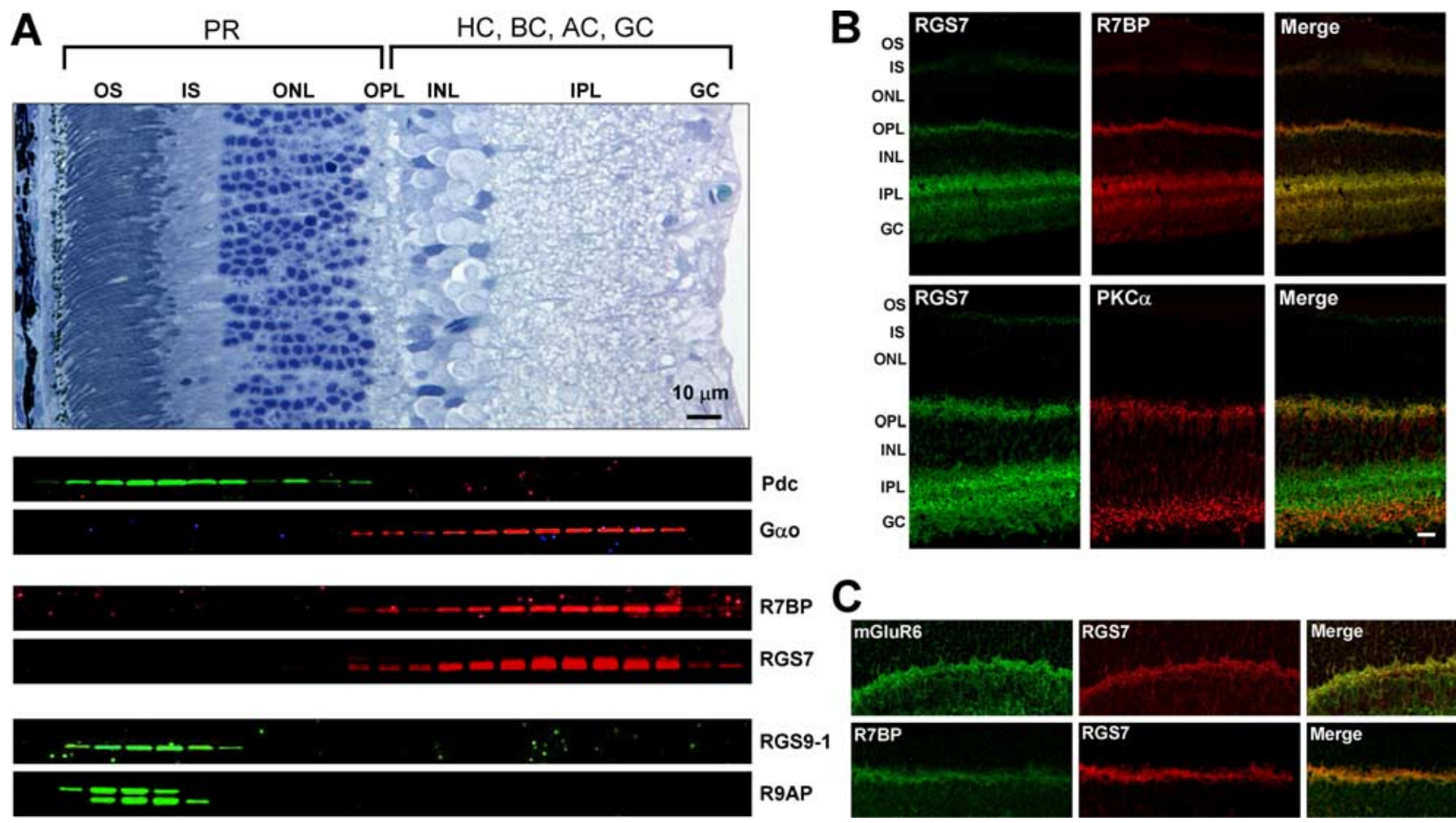

RGS9-1
R9AP
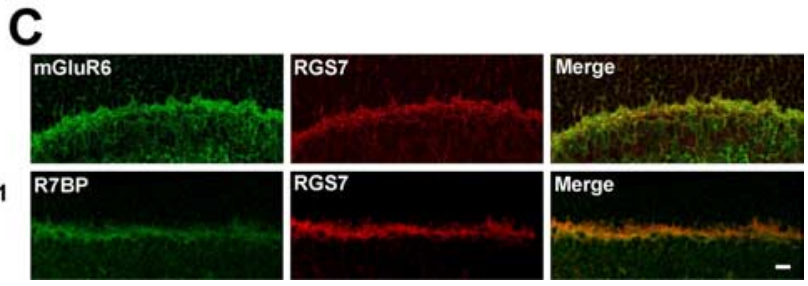

Figure 1. Colocalization of RGS7 and R7BP in the retina. A, Localization of R7BP and RGS7 as determined by a serial sectioning approach. Distribution of R7BP and RGS7 in $10 \mu \mathrm{m}$ sections from rat retina was compared with the localization of marker proteins: phosducin (Pdc) for photoreceptors and G-protein G $\alpha$ o for bipolar cells. Colocalization of RGS9-1 and R9AP proteins in photoreceptors was used as a reference. $\boldsymbol{B}$, Immunostaining of frozen retina cross sections with antibodies against RGS7 (green), R7BP (red), and bipolar cell marker PKC $\alpha$ (red). Merged images (yellow) reflect colocalization of proteins. Scale bar, $20 \mu \mathrm{m}$. C, High-magnification confocal images of the outer plexiform layer stained with the antibodies against RGS7 (red) and R7BP or mGluR6 (green). Merged channel (yellow) shows colocalization. Scale bar, $10 \mu \mathrm{m}$. The following abbreviations are used in this and subsequent figures: PR, photoreceptors; $\mathrm{HC}$, horizontal cells; $\mathrm{BC}$, bipolar cells, $\mathrm{AC}$, amacrine cells; GC, ganglion cells; OS, outer segment; IS, inner segment; ONL, outer nuclear layer; INL, inner nuclear layer; OPL, outer plexiform layer; IPL, inner plexiform layer.

2007b) and suggests that association with R7BP is not required for sustaining proteolytic stability of RGS7/G 35 complex.

We and others have previously reported that in the brain the stability of R7BP is reciprocally controlled by its association with R7 RGS proteins that protect it from proteolytic degradation (Anderson et al., 2007b; Grabowska et al., 2008). We have addressed the question of whether similar mechanisms control the expression of R7BP in the retina using $\mathrm{G} \beta 5-/-$ mice that lack all R7 RGS proteins. No detectable levels of R7BP were present in G $35-/-$ retinas (Fig. $2 C$ ), indicating that $\mathrm{R} 7 \mathrm{BP}$ in the retina exists only as a constitutive complex with R7 RGS proteins. We took advantage of this observation to determine the extent of R7BP interaction with RGS7 relative to RGS11, the only other R7 RGS protein reported to be localized in the bipolar cell dendrites and capable of interacting with R7BP (Morgans et al., 2007; Rao et al., 2007; Song et al., 2007). If a significant fraction of R7BP is bound to RGS11, then elimination of RGS11 is expected to result in a substantial reduction in R7BP levels. However, knock-out of RGS11 did not alter the expression levels of R7BP (Fig. 2C), suggesting that only a minor fraction of RGS11 is likely to exist as a complex with R7BP. A similar lack of effect on R7BP expression was observed in RGS9 - / - mice, consistent with the observation that RGS9 and R7BP exhibit essentially nonoverlapping localization.

In the process of characterizing RGS11 and G $\beta 5$ knock-outs, we have inadvertently discovered that rabbit anti-RGS11 CT antibodies used in the previous study (Song et al., 2007) recognized, in addition to RGS11, a nonspecific protein band migrating at the same position during gel electrophoresis. RGS11 antibodies raised against the same epitope in sheep (see Materials and Methods) were devoid of this artifact, as evidenced by the complete disappearance of RGS11 reactivity in both RGS11 and G $\beta 5$ knock-out mice (Fig. 2C). Since discovering a nonsignificant level of complex formation between RGS11 and R7BP, we revisited the binding of RGS11 to its second partner, R9AP, using the better-validated sheep antibodies. Unexpectedly, we find that, contrary to our previous report (Song et al., 2007), RGS11 was undetectable in the retinas of R9AP knock-outs, indicating that most of RGS11 is present in a complex with R9AP, which is required for ensuring its stability. Together, these data indicate that RGS7/G $\beta 5$ is the predominant binding partner of R7BP in ON-bipolar cells.

\section{Reduced plasma membrane association but unaltered} dendritic targeting of RGS7 in the absence of R7BP

Stable expression of RGS7/G $\beta 5$ in R7BP knock-outs made it possible to examine the role of R7BP in mediating subcellular targeting of the complex. Biochemical fractionation of retinal neurons revealed that the loss of R7BP results in a significant shift of RGS7 from the plasma membrane to cytoplasm (Fig. 3A), consistent with the role of R7BP as a membrane anchor. Quantification of the results indicated that $\mathrm{R} 7 \mathrm{BP}$ is responsible for membrane anchoring of $\sim 20 \%$ of RGS7 (Fig. $3 B$ ). To examine whether loss of this plasma membrane fraction has an effect on the targeting of RGS7 to the dendritic tips of bipolar cells, we studied its localization in $\mathrm{R} 7 \mathrm{BP}-/-$ retinas. Data presented in Figure $3 C$ reveal the preservation of the overall RGS7 immunoreactivity pattern in the absence of R7BP, including prominent staining in the outer plexiform layer. Immunostaining for $\mathrm{G} \beta 5$ also revealed no significant changes in the distribution between $\mathrm{R} 7 \mathrm{BP}-/-$ and wild-type retinas, suggesting the lack of unexpected anomalies in targeting R7 RGS proteins in the knock-out retinas. Detailed analysis of the 
outer plexiform layer region showed that R7BP knock-outs exhibit unaltered elaboration of ON-bipolar cell dendritic trees as well as robust punctate RGS7 immunoreactivity at the tips of the dendrites, suggesting preservation of normal synaptic connectivity between photoreceptors and bipolar cells (Fig. 3D). Counting of RGS7 puncta per PKC $\alpha$-positive ON-bipolar cells revealed equal numbers of these postsynaptic elements between R7BP knock-out and wild-type retinas: $9.9 \pm 0.7$ and $10 \pm 1.5$, respectively (SEM; $n=3$ counted per $4800 \mu \mathrm{m}^{2}$ area). These data argue that efficient targeting of RGS7 to the dendritic tips of ON-bipolar neurons occurs independently from its association with R7BP.

\section{Physiological responses of rod bipolar cells in R7BP knock-out mice}

Recent findings indicate that the loss of G $\beta 5$ subunit results in the disruption of synaptic transmission between photoreceptor and ON-bipolar cells (Rao et al., 2007). Our results suggest that $G \beta 5$ bound to RGS7 at this synapse exists predominantly as a complex with R7BP. It was therefore of interest to analyze whether R7BP is an essential component of G $\beta 5$ RGS complexes in mediating ON-bipolar cell responses to light. To test this possibility, we recorded ERG responses of R7BP knock-out mice, and found unaltered aand b-wave components, mostly indistinguishable from those of wild-type mice

(Fig. 4A). This suggests that, unlike in G $\beta 5$ knock-outs, the formation and function of the first visual synapse in R7BP knockouts is intact.

To examine in more detail the synaptic transmission between rods and ON-bipolar cells, we have performed whole-cell voltage-clamp recordings from rod ON-bipolar cells in darkadapted retinal slices after light excitation. Average response families from R7BP - / - rod bipolar cells closely resembled those of wild-type littermates (Fig. 4B). Furthermore, both the average response per photon calculated from the dimmest responses of each flash family (Fig. 4C) and the average relationship between flash strength and response amplitude (Fig. 4D) show little difference.

Overall, these data indicate that deletion of R7BP does not affect the formation and function of synapse between photoreceptor and ON-bipolar signal transmission, suggesting that RGS/ G $\beta 5$ complexes delivered to the postsynaptic sites independently from R7BP retain their functionality and preserve normal synaptic connectivity between photoreceptors.

\section{Discussion}

Many signaling proteins are differentially targeted to two major compartments of neuronal cells: axons and dendrites underpinning the functional polarization of the cells and suggesting the existence of specific mechanisms that ensure specific delivery of proteins to sites of their function (Arimura and Kaibuchi, 2007). Photoreceptors, because of their highly polarized nature, for many years have served as a convenient model to study principles of protein targeting (Papermaster, 2002; Deretic, 2006). In these neurons, components of the phototransduction cascade are specifically delivered to the dendritic specialization, the outer segment (Burns and Arshavsky, 2005). Among these proteins is the GTPase-activating complex RGS9-1/G $\beta 5 /$ R9AP, which relies on R9AP subunit for the intracellular targeting (Martemyanov et al., 2003; Keresztes et al., 2004). Similar targeting principles appear to be conserved in the brain, where the R9AP homolog, R7BP is responsible for the localization of RGS9-2/G $\beta 5$ to another dendritic destination, the postsynaptic density (Anderson et al., 2007a,b). Conservation of the mechanisms suggested a trimeric model for targeting of RGS-G $\beta 5$ complexes by the adapter subunits R9AP or R7BP.

All members of the R7 RGS family can form complexes with the targeting subunit, R7BP (Drenan et al., 2005; Martemyanov et al., 2005). These complexes are expressed in many types of neurons and regulate fundamental processes that in addition to vision include movement control, reward processing, and nociception (Garzón et al., 2003; Rahman et al., 2003; SánchezBlázquez et al., 2003). Subcellular localization of R7 RGS proteins was established best in the retinal bipolar cells, revealing their prominent postsynaptic targeting in the dendrites (Morgans et al., 2007; Rao et al., 2007). In our study, we tested the hypothesis that all R7 RGS/G $\beta 5$ complexes require R7BP for mediating their postsynaptic targeting. Surprisingly, our results indicate the existence of the alternative, R7BP-independent, mechanisms divid- 

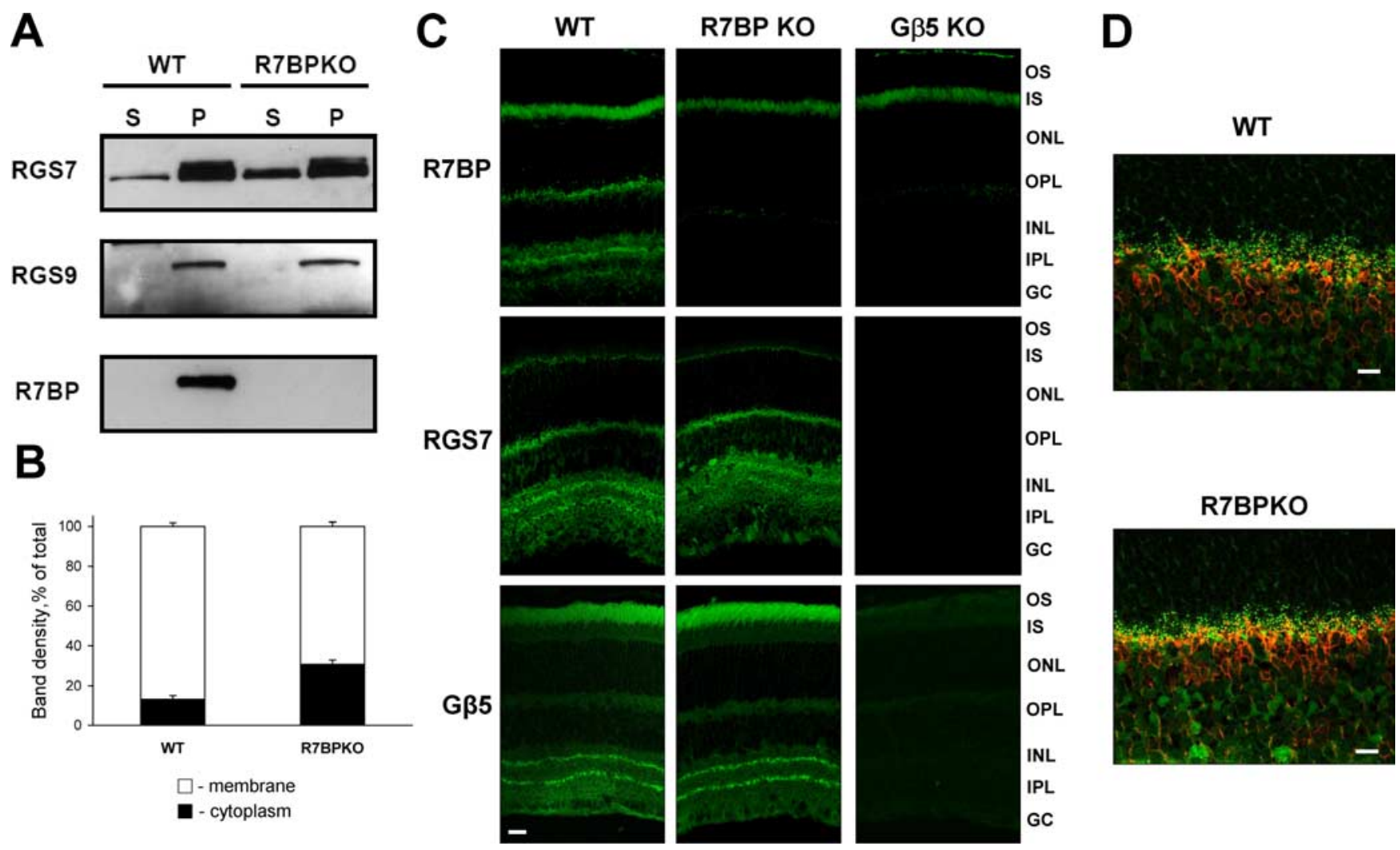

Figure 3. Localization of RGS7/G $\beta 5$ in R7BP knock-out retinas. $A$, Subcellular fractionation shows shift of RGS7 to cytoplasmic fraction in R7BP knock-out retinas. Retina lysates were separated into soluble cytoplasmic (S) and insoluble membrane (P) compartments by centrifugation. The presence of proteins in each fraction was revealed by Western blotting. $\boldsymbol{B}$, Quantification of data presented in $\boldsymbol{A}$ from three independent experiments. RGS7 band intensities were determined by densitometry using ImageJ software (NIH) and plotted as percentage of total density. Error bars indicate SEM. C, Analysis of RGS7 and G $\beta 5$ localization in retina cross sections by immunostaining. Sections from G $\beta 5$ and R7BP knock-outs were used as a control for nonspecific staining. Scale bar, $20 \mu \mathrm{m}$. D, High-power images of RGS7 immunoreactivity (green) in synapses of ON-bipolar cells stained with anti-PKC $\alpha$ antibodies (red). Distinct green puncta correspond to RGS7 enriched in dendritic tips of bipolar cells. Scale bar, $10 \mu \mathrm{m}$. K0, Knock-out; WT, wild type.

ing R7 RGS complexes into two groups: obligatory trimers

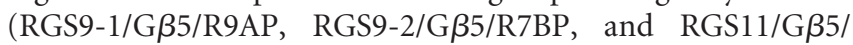
R9AP) and dimeric complexes whose targeting, expression, and function is independent of R9AP/R7BP (RGS7/G 35 and RGS6/ G $\beta 5$ ). Curiously, sequence homology analysis reveals a similar segregation pattern in which RGS9 and RGS11 form one cluster, and RGS6 and RGS7 form another (Sierra et al., 2002), suggesting that reliance on targeting subunits might have developed after evolutionary specification of the two subgroups of R7 RGS proteins.

Our study suggests that in contrast to RGS9/11, RGS6/7 possess unique elements that mediate its R9AP/R7BP-independent targeting. Because in the absence of interaction with R7BP, the membrane localization of a majority of the RGS7 is preserved, it is possible that its subcellular targeting might be mediated by other membrane proteins. In this light, it is intriguing to speculate that RGS7/G $\beta 5$ targeting might be directly mediated by mGluR6, because G-protein-coupled receptors were recently shown to bind structural elements present in the R7 RGS proteins (Ballon et al., 2006).

Recent studies have established an essential role of R7 RGS/ G $\beta 5$ complexes in mediating synaptic transmission between photoreceptors and bipolar cells, because inner retinal neurons of G $\beta 5$ knock-out mice lacking all R7 RGS/G $\beta 5 /$ R7BP complexes are unable to respond to light stimulation (Rao et al., 2007). Although it is not known whether RGS/G $\beta 5$ protein complexes are involved in synaptogenesis or are critical for the mGluR6-
G $\alpha$ o signaling in the ON-bipolar neurons, it was established that the postsynaptic action of the complexes downstream from the photoreceptors is required (Rao et al., 2007). By comparing expression and localization of R7 RGS complexes in the retinas of several mouse knock-out lines, we can narrow down the identity of the complexes essential for the synaptic transmission to RGS7/ G $\beta 5$ or RGS6/G $\beta 5$ dimers. Knock-outs of RGS9 (Lyubarsky et al., 2001), RGS11 (Mojumder and Wensel, 2008), or R9AP (Jeffrey et al., 2007), which lack RGS9-1/G $\beta 5 / R 9 A P, R G S 11 / G \beta 5 /$ $\mathrm{R} 9 \mathrm{AP}$, or both complexes, display robust b-wave upon ERG analysis. This indicates that normal synaptic transmission is preserved, and rules out these complexes from contributing to synaptic deficiency observed in the G $\beta 5$ knock-out. Furthermore, we found that the elimination of R7BP did not affect the kinetics of ON-bipolar responses to light, excluding one more component of the trimeric complexes. Although we would like to speculate that intact synaptic transmission requires mainly the RGS7/G $\beta 5$ dimer, because it appears to be enriched in the bipolar cells over RGS6/G $\beta 5$, which is mainly present in starburst amacrine cells (Song et al., 2007), the high homology of these complexes and the lack of specific antibodies that can fully discriminate between RGS6 and RGS7 make this an open question that should be addressed with individual knock-outs of either RGS6 or RGS7. Likewise, elucidating the mechanisms of R7BPindependent dendritic targeting of RGS6/7 will also be an exciting future direction, because it could potentially explain how these protein(s) regulate synaptic transmission. 

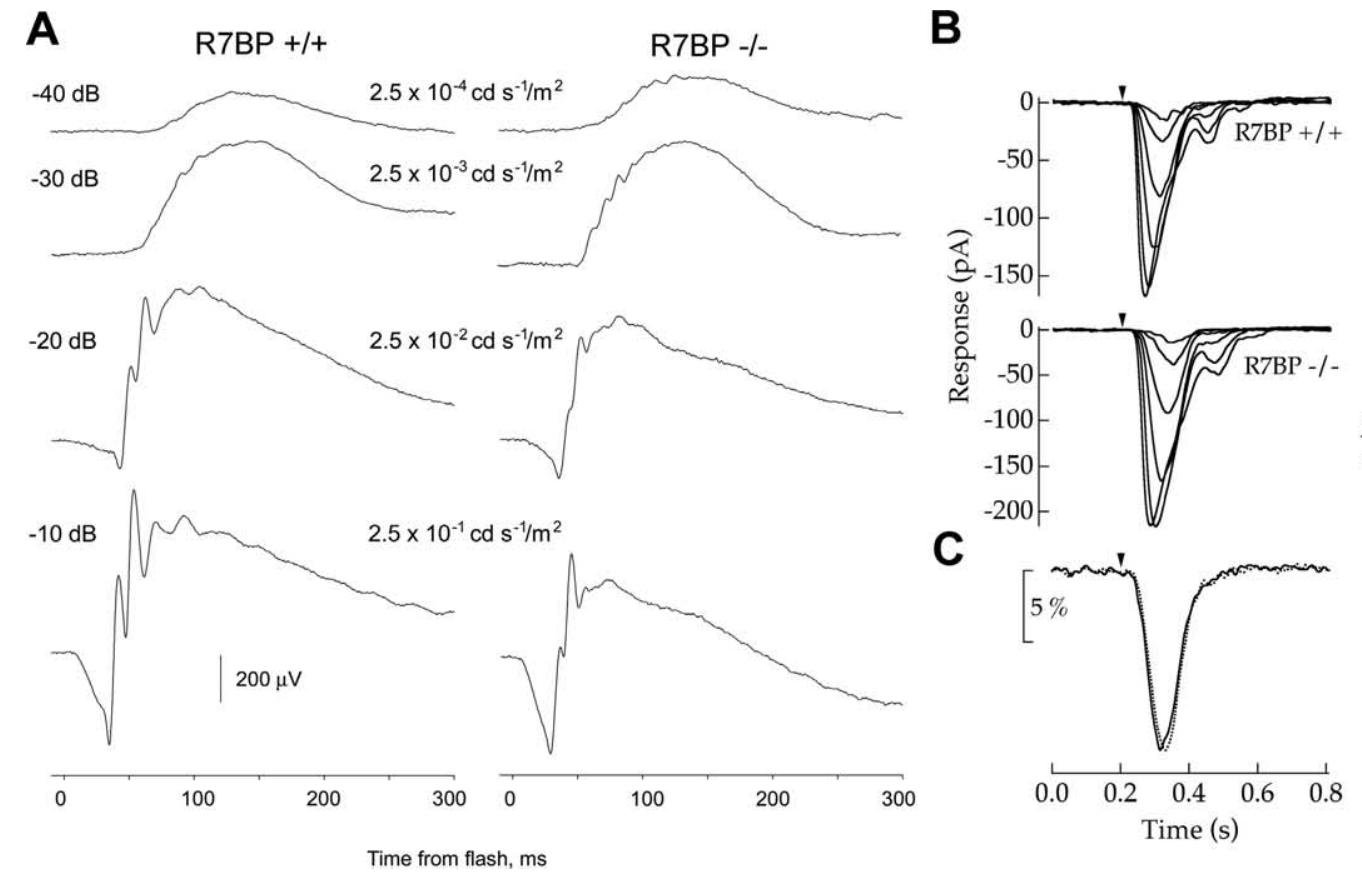

D

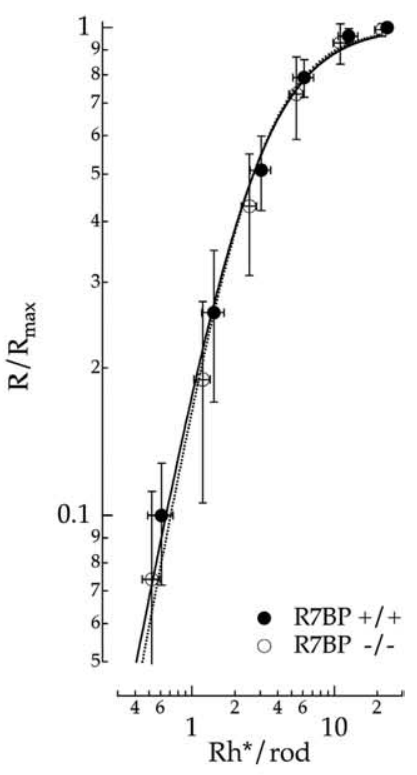

Figure 4. Light-evoked responses of R7BP KO retinas. $\boldsymbol{A}-\boldsymbol{D}$, Electrophysiological responses were analyzed by ERG $(\boldsymbol{A})$ and single-cell voltage-clamp recordings from $0 \mathrm{~N}$-bipolar cells ( $\boldsymbol{B}-\boldsymbol{D})$. $\boldsymbol{A}$, ERGs from wild type (R7BP $+1+$ ) and R7BP $-/-$ mice. Shown are typical responses evoked by $-40,-30,-20$, and $-10 \mathrm{~dB}$ UTAS-E400 flashes corresponding to the indicated illuminance in candelas per second per square meter. Traces were obtained using $300 \mathrm{~Hz}$ high-cut filter setting and the averaging of $5-10$ records. $\boldsymbol{B}$, Light-evoked inward currents in rod bipolar cells were plotted as a function of time for brief flashes (arrowheads) of $0.52,1.2,2.7,5.4,11$, and $22 \mathrm{Rh} /$ rod. The bandwidth was $50 \mathrm{~Hz}$. C, Estimated responses per photon plotted as a percentage of maximum inward current. The R7BP + / response (solid) was the average of 244 responses across 14 rod bipolar cells resulting from a flash that on average generated $0.9 \mathrm{Rh} / \mathrm{rod}$. The R7BP $-/-$ response (dotted) was the average of 211 responses across 16 rod bipolar cells resulting from a flash that on average also generated $0.9 \mathrm{Rh}^{*} /$ rod. $\boldsymbol{D}$, Response-intensity relationship across all rod bipolar cells in $\mathrm{R} 7 \mathrm{BP}+/+(n=14)$ and R7BP $-/-(n=16)$ mice. Data are plotted as mean \pm SD, and the least-squares Hill curve fits to the mean and SDs are superimposed. For R7BP $+/+$ rod bipolar cells, $I_{1 / 2}=2.7 \mathrm{Rh}^{*} / \mathrm{rod}$, and $n=1.56$ (solid). For R7BP $-/-$ rod bipolar cells, $I_{1 / 2}=2.7 \mathrm{Rh}^{*} / \mathrm{rod}$, and $n=1.64$ (dotted).

\section{References}

Anderson GR, Semenov A, Song JH, Martemyanov KA (2007a) The membrane anchor R7BP controls the proteolytic stability of the striatal specific RGS protein, RGS9-2. J Biol Chem 282:4772-4781.

Anderson GR, Lujan R, Semenov A, Pravetoni M, Posokhova EN, Song JH, Uversky V, Chen CK, Wickman K, Martemyanov KA (2007b) Expression and localization of RGS9-2/G 5/R7BP complex in vivo is set by dynamic control of its constitutive degradation by cellular cysteine proteases. J Neurosci 27:14117-14127.

Arimura N, Kaibuchi K (2007) Neuronal polarity: from extracellular signals to intracellular mechanisms. Nat Rev Neurosci 8:194-205.

Ballon DR, Flanary PL, Gladue DP, Konopka JB, Dohlman HG, Thorner J (2006) DEP-domain-mediated regulation of GPCR signaling responses. Cell 126:1079-1093.

Burns ME, Arshavsky VY (2005) Beyond counting photons: trials and trends in vertebrate visual transduction. Neuron 48:387-401.

Chen CK (2005) The vertebrate phototransduction cascade: amplification and termination mechanisms. Rev Physiol Biochem Pharmacol 154:101-121.

Chen CK, Eversole-Cire P, Zhang H, Mancino V, Chen YJ, He W, Wensel TG, Simon MI (2003) Instability of GGL domain-containing RGS proteins in mice lacking the $G$ protein $\beta$-subunit G $\beta 5$. Proc Natl Acad Sci U S A 100:6604-6609.

Deretic D (2006) A role for rhodopsin in a signal transduction cascade that regulates membrane trafficking and photoreceptor polarity. Vis Res 46:4427-4433.

Dhingra A, Lyubarsky A, Jiang M, Pugh EN Jr, Birnbaumer L, Sterling P, Vardi N (2000) The light response of ON bipolar neurons requires G $\alpha$ o. J Neurosci 20:9053-9058.

Drenan RM, Doupnik CA, Boyle MP, Muglia LJ, Huettner JE, Linder ME, Blumer KJ (2005) Palmitoylation regulates plasma membrane-nuclear shuttling of R7BP, a novel membrane anchor for the RGS7 family. J Cell Biol 169:623-633.

Field GD, Rieke F (2002) Nonlinear signal transfer from mouse rods to bipolar cells and implications for visual sensitivity. Neuron 34:773-785.
Garzón J, López-Fando A, Sánchez-Blázquez P (2003) The R7 subfamily of RGS proteins assists tachyphylaxis and acute tolerance at mu-opioid receptors. Neuropsychopharmacology 28:1983-1990.

Grabowska D, Jayaraman M, Kaltenbronn KM, Sandiford SL, Wang Q, Jenkins S, Slepak VZ, Smith Y, Blumer KJ (2008) Postnatal induction and localization of R7BP, a membrane-anchoring protein for regulator of $\mathrm{G}$ protein signaling 7 family-Gbeta5 complexes in brain. Neuroscience 151:969-982.

Jeffrey BG, Morgans CW, Duvoisin RM (2007) R9AP modulates activation of the bipolar cell response as measured in vivo with the ERG. Invest Ophthalmol Vis Sci 48:E-4185.

Keresztes G, Martemyanov KA, Krispel CM, Mutai H, Yoo PJ, Maison SF, Burns ME, Arshavsky VY, Heller S (2004) Absence of the RGS9/Gbeta5 GTPase-activating complex in photoreceptors of the R9AP knockout mouse. J Biol Chem 279:1581-1584.

Krispel CM, Chen D, Melling N, Chen YJ, Martemyanov KA, Quillinan N, Arshavsky VY, Wensel TG, Chen CK, Burns ME (2006) RGS expression rate-limits recovery of rod photoresponses. Neuron 51:409-416.

Lyubarsky AL, Naarendorp F, Zhang X, Wensel T, Simon MI, Pugh EN Jr (2001) RGS9-1 is required for normal inactivation of mouse cone phototransduction. Mol Vis 7:71-78.

Makino ER, Handy JW, Li TS, Arshavsky VY (1999) The GTPase activating factor for transducin in rod photoreceptors is the complex between RGS9 and type 5 G protein $\beta$-subunit. Proc Natl Acad Sci U S A 96:1947-1952.

Martemyanov KA, Lishko PV, Calero N, Keresztes G, Sokolov M, Strissel KJ, Leskov IB, Hopp JA, Kolesnikov AV, Chen CK, Lem J, Heller S, Burns ME, Arshavsky VY (2003) The DEP domain determines subcellular targeting of the GTPase activating protein RGS9 in vivo. J Neurosci 23:10175-10181.

Martemyanov KA, Yoo PJ, Skiba NP, Arshavsky VY (2005) R7BP, a novel neuronal protein interacting with RGS proteins of the R7 family. J Biol Chem 280:5133-5136.

Mojumder DK, Wensel TG (2008) Variable distribution of RGS7 and RGS11 in the dendritic-tips of ON-bipolar cells in the murine retina. Invest Ophthalmol Vis Sci 49:E-762. 
Morgans CW, Wensel TG, Brown RL, Perez-Leon JA, Bearnot B, Duvoisin RM (2007) Gbeta5-RGS complexes co-localize with mGluR6 in retinal ON-bipolar cells. Eur J Neurosci 26:2899-2905.

Nini L, Waheed AA, Panicker LM, Czapiga M, Zhang JH, Simonds WF (2007) R7-binding protein targets the G protein beta 5/R7-regulator of G protein signaling complex to lipid rafts in neuronal cells and brain. BMC Biochem 8:18.

Papermaster DS (2002) The birth and death of photoreceptors: the Friedenwald Lecture. Invest Ophthalmol Vis Sci 43:1300-1309.

Rahman Z, Schwarz J, Gold SJ, Zachariou V, Wein MN, Choi KH, Kovoor A, Chen CK, DiLeone RJ, Schwarz SC, Selley DE, Sim-Selley LJ, Barrot M, Luedtke RR, Self D, Neve RL, Lester HA, Simon MI, Nestler EJ (2003) RGS9 modulates dopamine signaling in the basal ganglia. Neuron 38:941-952.

Rao A, Dallman R, Henderson S, Chen CK (2007) G $\beta 5$ is required for normal light responses and morphology of retinal ON-bipolar cells. J Neurosci 27:14199-14204.

Rodieck RW (1998) The first steps in seeing. Sunderland, MA: Sinauer.

Sampath AP, Strissel KJ, Elias R, Arshavsky VY, McGinnis JF, Chen J,
Kawamura S, Rieke F, Hurley JB (2005) Recoverin improves rodmediated vision by enhancing signal transmission in the mouse retina. Neuron 46:413-420.

Sánchez-Blázquez P, Rodríguez-Díaz M, López-Fando A, Rodríguez-Muñoz M, Garzón J (2003) The GBeta5 subunit that associates with the R7 subfamily of RGS proteins regulates mu-opioid effects. Neuropharmacology 45:82-95.

Sierra DA, Gilbert DJ, Householder D, Grishin NV, Yu K, Ukidwe P, Barker SA, He W, Wensel TG, Otero G, Brown G, Copeland NG, Jenkins NA, Wilkie TM (2002) Evolution of the regulators of G-protein signaling multigene family in mouse and human. Genomics 79:177-185.

Sokolov M, Strissel KJ, Leskov IB, Michaud NA, Govardovskii VI, Arshavsky VY (2004) Phosducin facilitates light-driven transducin translocation in rod photoreceptors. Evidence from the phosducin knockout mouse. J Biol Chem 279:19149-19156.

Song JH, Song H, Wensel TG, Sokolov M, Martemyanov KA (2007) Localization and differential interaction of R7 RGS proteins with their membrane anchors R7BP and R9AP in neurons of vertebrate retina. Mol Cell Neurosci 35:311-319. 